\title{
Complete band gaps in two-dimensional photonic quasicrystals
}

\author{
Marian Florescu, ${ }^{1, *}$ Salvatore Torquato, ${ }^{2}$ and Paul J. Steinhardt ${ }^{2}$ \\ ${ }^{1}$ Department of Physics, Princeton University, Princeton, New Jersey 08544, USA \\ ${ }^{2}$ Department of Chemistry, Princeton University, Princeton, New Jersey 08544, USA \\ and Princeton Center for Theoretical Sciences, Princeton University, Princeton, New Jersey 08544, USA
}

(Received 1 August 2009; revised manuscript received 11 September 2009; published 6 October 2009)

\begin{abstract}
We introduce an optimization method to design examples of photonic quasicrystals with substantial, complete photonic band gaps; that is, a range of frequencies over which electromagnetic wave propagation is forbidden for all directions and polarizations. The method can be applied to photonic quasicrystals with arbitrary rotational symmetry; here, we illustrate the results for fivefold and eightfold symmetric quasicrystals. The optimized band gaps are highly isotropic, which may offer advantages over photonic crystals for certain applications.
\end{abstract}

DOI: 10.1103/PhysRevB.80.155112

PACS number(s): 61.44.Br, 41.20.Jb, 42.70.Qs, 78.66.Vs

\section{INTRODUCTION}

Photonic materials with complete photonic band gaps (PBGs), i.e., frequency ranges over which electromagnetic wave propagation is prohibited for all directions and polarizations, are artificial dielectric heterostructures that enable control of the generation and flow of light. Their development ${ }^{1,2}$ has evolved dramatically in the last decade and their unusual properties have found a wide range of applications including efficient radiation sources, ${ }^{3}$ telecommunications devices, ${ }^{4}$ sensors, ${ }^{5}$ and optical computer chips. ${ }^{6}$ Until recently, the only known materials with complete PBGs were photonic crystals comprised of a periodic arrangements of dielectric materials. In photonic crystals, the opening of the PBG is understood to be governed by the synergetic interplay between Bragg scattering resonances of the periodic dielectric array and the Mie resonances of individual dielectric scattering centers. ${ }^{7}$

A similar combination of scattering mechanisms is possible in photonic quasicrystals, in which the dielectric materials are arranged in a pattern with long-range quasiperiodic translational order and rotational symmetries forbidden to crystals (such as fivefold symmetry in two dimensions and icosahedral symmetry in three dimensions) ${ }^{8}$ Quasiperiodic order also produces Bragg scattering. ${ }^{8}$ In fact, there have been numerous studies of photonic quasicrystals with PBGs in the literature. ${ }^{9-16}$ Their band gaps are found to be considerably more isotropic and, for two-dimensional quasicrystals, the dielectric contrast required to open the band gaps for TM polarization (electric field oscillating out of the plane) is smaller than the contrast required for the periodic counterparts. ${ }^{10}$ Most of the photonic quasicrystalline structures considered only have band gaps for either TM- or TEpolarized radiation. ${ }^{9,13,15}$ Two examples of complete band gaps have been found previously; ${ }^{12,16}$ here we present a systematic method to produce substantially greater fundamental complete band gaps for photonic quasicrystals with arbitrary symmetry.

In general, the architectures required for optimal TM band gaps are quite different than optimal architectures for TE polarization (electric field oscillating in the plane). Structures based on a distribution of isolated dielectric inclusions (e.g., rods) is best for TM band gaps while structures based on connected dielectric networks are optimal for TE. ${ }^{17}$ For the case of quasicrystalline systems, with their rich variety of local environments, the problem of finding an optimal compromise becomes more difficult. Consequently, until now, there has not been a systematic procedure for designing quasiperiodic photonic structures with sizable PBGs for all directions of propagation and polarizations.

In this paper, we introduce an optimization scheme discussed in Ref. 18 (where it was applied to disordered heterostructures) to design two-dimensional photonic quasicrystals with substantial complete PBGs, comparable to the largest band gaps found for photonic crystals. The examples illustrated here are based on the vertices of a Penrose tiling, ${ }^{19}$ a fivefold symmetric pattern composed of obtuse and acute rhombi and on the vertices of an octagonal tiling, ${ }^{20}$ an eightfold symmetric pattern composed of squares and rhombi.

In Sec. II, we describe how the quasiperiodic patterns are constructed. In Sec. III, we present the results of the bandstructure calculations and analyze the properties of the resulting PBGs including the field distribution of the photonic modes that define the photonic band edges. Section IV provides concluding remarks.

\section{NEARLY OPTIMAL PHOTONIC BAND-GAP STRUCTURES FROM QUASIPERIODIC POINT PATTERNS}

The Penrose and octagonal quasicrystalline point patterns considered in this work are constructed by the projection and cut method, ${ }^{21}$ which consists of projecting the points of an $n$-dimensional hypercubic lattice into the three-dimensional space, where $n=4$ for the octagonal lattice and $n=5$ for the Penrose lattice. In general, the lack of spatial periodicity makes it impossible to define a unit cell for band-gap calculations; we address this by using a series of rational periodic approximants of the quasicrystal. In the case of the Penrose lattice periodic approximants are constructed by the same projection method by replacing by a Fibonacci ratio $\left(\tau_{n}\right.$ $\left.=p_{n} / q_{n}=1 / 1,2 / 1,3 / 2,5 / 3, \ldots\right)$. The unit cell is rectangularly shaped and its area grows as $n$ increases and the rational approximant approaches $\tau$. In particular, for a rational ap- 
proximant $\tau_{n}=p_{n} / q_{n}$, the unit cell has dimensions $L_{x}=5\left[p_{n}\right.$ $+q_{n}(\tau-1), L_{y}=\sqrt{3-\tau}\left(p_{n} \tau+q_{n}\right)$ and contains $N=10 p_{n}(p$ $+2 q_{n}$ ) vertices of the rhombic pattern. In the case of the octagonal quasilattices, we replace by its continuous fraction series $\left(\rho_{n}=p_{n} / q_{n}=1 / 1,3 / 2,7 / 5, \ldots\right)$ and the resulting periodic approximant has a square-shaped unit cell of side $L$ $=p_{n}+\rho q_{n}$ containing $N=\left[p_{n}+\left(\rho^{2}+\rho\right) q_{n}\right]\left[p_{n}+\left(\rho^{2}-\rho\right) q_{n}\right]$ vertices of the square-rhombic pattern. The periodic approximants constructed this way results in tilings consisting of identical tiles as the original quasiperiodic tiling and transition from quasiperiodicity to periodicity is accommodated through the flipping of a certain number of tiles. These rational periodic approximants are known to be the best approximants of the quasiperiodic lattice as they achieve the selected periodicity by introducing the minimum density of defects with respect to the perfect quasiperiodic tiling. ${ }^{22}$ The vertices of the tilings obtained by this method form a sequence of quasicrystal approximant point pattern.

The next step is to find the arrangement of dielectric material around the point pattern that produces the optimal TM, TE, and complete PBGs. Identifying these optimal dielectric distributions is well known to be a daunting computational task, despite the recent development of rigorous mathematical optimization methods. ${ }^{23-25}$

For the case of TM radiation only, a nearly optimal decoration for a given point pattern is obtained by placing identical dielectric cylinders centered at each point and adjusting the radius. For the optimal radius, there is a coincidence of Mie and Bragg scattering effects that lead to substantial band gaps. $^{7}$ The dielectric cylinders support Mie scattering resonances and, for frequencies above the lowest-order resonance, the scattered radiation is out of phase with the incident wave. This destructive interference prohibits radiation propagation and favors the opening of a complete TM gap. ${ }^{15}$ At the same time, the quasiperiodic long-range order in quasicrystalline systems results in a dense (no minimum separation) collection of Bragg peaks; however, it is notable that most of Bragg-peak intensities are infinitesimally small. The first set of intense Bragg peaks is associated with scattering on planes separated by the average distance in the quasicrystalline point pattern and can be employed to define an effective Brillouin zone. Therefore, as for photonic crystals, Bragg scattering also contributes to the PBG formation in photonic quasicrystals such that, whenever the wave vector of the incident radiation is directed along the effective Brillouin-zone boundary, the reflected and refracted waves interfere to cancel the incoming wave and prevent its propagation inside the structure. An optimal band gap occurs when the two scattering mechanism reinforce each other on a given spectral range. This approach can be applied to find near optimal TM band gap for the fivefold and eightfold quasicrystal patterns obtained by projection. ${ }^{25}$

To obtain the optimal PBGs for TE radiation, where the electric field is oriented in the plane of the scatters, necessitates a different dielectric arrangement. Instead of isolated cylinders, a connected network of dielectric with air pockets in between is favored. For example, a commonly used configuration for photonic crystals is "inverted" compared to the optimal structures for TM PBGs, i.e., placing an identical air cylinder at each point so as to produce a connected network

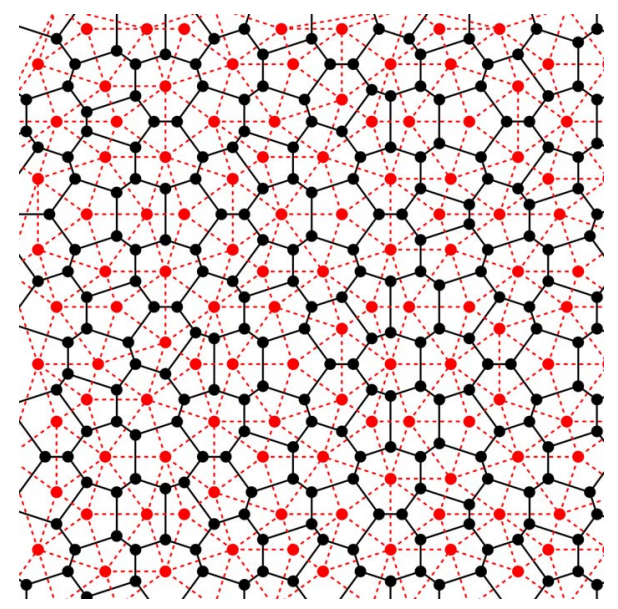

FIG. 1. (Color online) Protocol described in Sec. II maps the vertices of a rhombus tiling (red/gray points) into a network of cells whose vertices are trivalent (black segments and points). To construct a photonic material with a complete band gap, edges are replaced by a wall of dielectric of finite thickness and the vertices are replaced by cylinders of finite radius.

of dielectric material. However, in the case of quasicrystalline structures, we find this method fails to produce sizable fundamental TE PBGs. The main reason is that the inverted structure has a very nonuniform distribution of dielectric scattering regions that broadens the distribution of resonances. Similarly, placing walls along the edges of each Penrose tile as in Ref. 9 has rather nonuniform connectivity and fails to produce an optimal TE PBG.

To overcome this problem, we employ a protocol introduced in Ref. 18 that converts a general point pattern into photonic heterostructures with sizeable, nearly optimal TE PBGs. We briefly review the protocol here: consider an arbitrary point pattern denoted by red/gray disks in Fig. 1. In the example in the figure, the point pattern is enclosed in a square domain with periodic boundary conditions. First, we perform a Delaunay tessellation of the original point pattern. This provides a triangular partitioning that minimizes the standard deviation of the triangle angles around the mean of $\pi / 6$. Next, associated with each triangle is a centroidal point; these are connected with line segments to form cells around the original points. The nearly optimal heterostructure for TE band gaps is obtained by decorating the edges of the cell network with walls (along the azimuthal direction) of dielectric material of uniform width $w$, as displayed in Fig. 1. We note that this procedure is universal and can be used to generate connected network architectures based on periodic, quasiperiodic, and disordered point patterns. Finally, the optimal structure is obtained by varying a single parameter, the cell thickness, and identifying the value that maximizes the TE band gap.

The total number of cells in the network is the same as the number of points in the original point pattern and each vertex of the network is trivalent. The cells can be regarded as individual scattering objects supporting electromagnetic resonances, which become coupled to each other when placed into the connected network.

With an additional step, the protocol can be used to a 


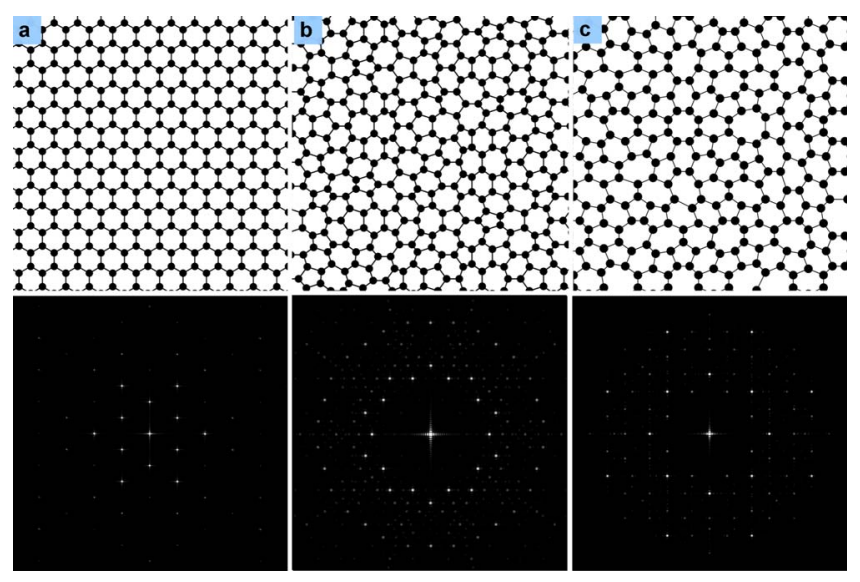

FIG. 2. (Color online) Optimal photonic crystal and quasicrystal heterostructures and their diffraction patterns derived from (a) a periodic sixfold symmetric point pattern; (b) a fivefold symmetric quasicrystal (Penrose) tiling; and (c) an eightfold symmetric quasicrystal tiling.

heterostructure with a nearly optimal complete PBG, i.e., maximal overlap between TM and TE gaps. Namely, each vertex of the trihedral network constructed above is decorated with a cylinder or radius $r$. The number of cylinder is equal to twice the number of cells. The optimal complete band gap obtained by varying the two free parameters, the cell wall thickness $w$ and cylinder radius $r$. As shown in Fig. 2 , the Fourier spectrum of the decorated photonic structures obtained through this protocol fully preserve the symmetries of the original point pattern. In comparison, alternative designs based on inverted architectures or decorations of the quasicrystal tile edges with dielectric walls and its vertices with dielectric cylinders fails to open sizable complete PBGs.

\section{PHOTONIC BAND-GAP RESULTS}

We compute the photonic band structures for the optimal heterostructures (described in detail below) using a conventional plane-wave expansion formalism. ${ }^{26}$ For this approach, which assumes a periodic structure, we use a sequence of increasingly accurate periodic approximants and check for convergence. For the purposes of illustration, we assume the photonic materials are composed of silicon (with dielectric constant $\epsilon=11.56$ ) and air. In all the numerical simulations, we use $32^{2} \times N_{P}$ plane waves to achieve convergence accuracy of better than $1 \%$ for the lowest $N_{P}$ photonic bands. Here $N_{P}$ represents the number of scattering centers (TM case), cells (TE case) or centers and cells (TM+TE case). Most of the band-structure calculations are preformed around a contour along the first Brillouin zone of the respective system, which includes the high-symmetry $k$-space points, $\boldsymbol{\Gamma}=0, \mathbf{X}=\mathbf{b}_{1} / 2, \mathbf{M}=\left(\mathbf{b}_{1}+\mathbf{b}_{2}\right) / 2$, and $\mathbf{Y}=\mathbf{b}_{2} / 2$, where $\mathbf{b}_{1}$ and $\mathbf{b}_{2}$ are the basis vectors of the reciprocal lattice considered.

TM Band Gaps. To obtain optimal TM PBGs for a given symmetry, dielectric cylinders are placed at the vertices of the tilings obtained by projection.

For example, an optimized fivefold symmetric quasicrystalline pattern derived using the protocol from a $n=5 / 3$ pe-
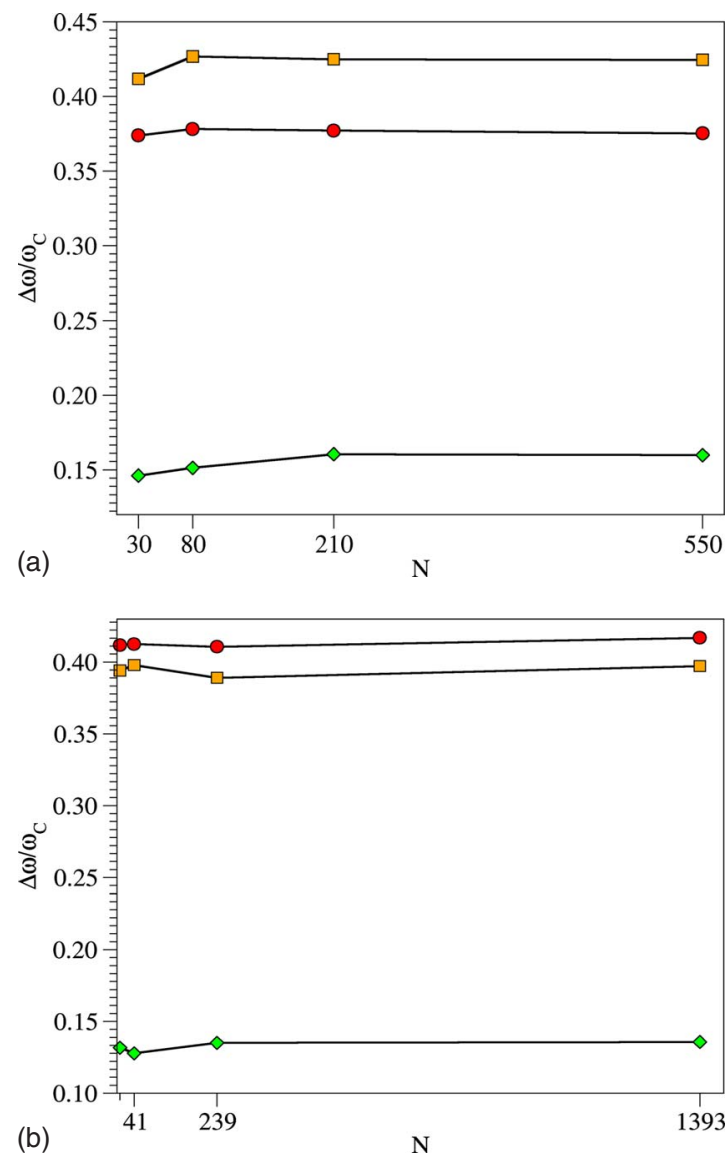

FIG. 3. (Color online) The fractional PBG width for (a) fivefold symmetric and (b) eightfold symmetric photonic quasicrystal as a function of the number of particles in the periodic approximant for the optimal TM (red circles), TE (orange squares), and complete (green diamonds) band gaps.

riodic approximant of the Penrose pattern displays a TM PBG of $\Delta \omega / \omega_{C}=39 \%$. An analogous optimized structure based on the $n=7 / 5$ periodic approximant of the octagonal lattice displays a TM PBG of $\Delta \omega / \omega_{C}=42 \%$, where $\omega_{C}$ is the gap central frequency. The optimal radius of the cylindrical inclusion is $r / a=0.177$ for the Penrose tiling and $r / a$ $=0.189$ for the octagonal lattice, were $a$ is the side length of the rhombic tiles obtained by projection. As a check, we perform a convergence analysis as the order of the periodic approximant increases; as shown in Fig. 3, the results are fully convergent.

We find that the band gap for the optimal structures always occurs between bands $N_{P}$ and $N_{P}+1$ where $N_{P}$ is the number of points in the periodic approximant point pattern. This is an analogous behavior to the band folding that occurs in periodic structures when analyzed with a supercell approach.

TE Band Gaps. The optimal TE band-gap designs are obtained by the protocol described in Sec. II. They correspond to the network of walls connecting the centroids of the Delaunay tiling. In the case of the fivefold symmetric quasicrystal, the optimum has wall width $w / a=0.103$; the TE band gap is $\Delta \omega / \omega_{C}=42.3 \%$, the largest ever reported for a photonic quasicrystal. For the octagonal quasicrystal, the optimal 
structure has dielectric material width $w / a=0.106$ along each edge, which produces a TE band gap of $\Delta \omega / \omega_{C}$ $=39.2 \%$.

The TE band-gap formation is analogous to the TM case in that it involves an interplay between scattering from individual cells and the Bragg scattering of the quasiperiodic arrangement of scattering planes. Similar to the case of TMpolarized radiation, we also obtain that the band gap for the optimal structures always occurs between bands $N_{P}$ and $N_{P}$ +1 where $N_{P}$ is now the number of cells in the structure.

Complete Band Gap. For the complete band gap, we find that the optimal structure consists of placing a wall along each edge and a cylinder at each trihedral vertex of the network generated by the protocol in Sec. II. The optimum for the fivefold symmetric case has cylinder radius is $r / a$ $=0.157$ and cell wall thickness $w / a=0.042$. For the eightfold symmetric point patterns, the optimum is $r / a=0.167$ and $w / a=0.014$. The scattering properties of the individual scattering centers and cells are again essential in the band-gap opening and the complete band gap occurs always between bands $3 N_{P}$ and $3 N_{P}+1$, where $N_{P}$ is the number of points in the periodic approximant point pattern (there are $3 N_{P}$ total scattering units in the system, $2 N_{P}$ dielectric disks, and $N_{P}$ dielectric cells).

The resulting optimal fivefold symmetric structure displays complete (TM and TE) PBG of $16.5 \%$ - the first complete band gap ever reported for a photonic quasicrystal with fivefold symmetry and comparable to the largest band gap (20\%) found for photonic crystals with the same dielectric contrast. $^{27}$ The optimal eightfold symmetric structure has a full PBG of $13.5 \%$.

As shown in Fig. 4, the band-gap size obtained by considering a contour along the Brillouin zone is completely consistent with the density-of-states calculations. Similar to the case of the TM and TE PBGs, the system-size study presented in Fig. 3 reveals that the band-gap size becomes independent of the size of the periodic approximant when $N_{P} \geq 100$ (with $N_{P}$ the number of points in the original point pattern), implying that our results have converged to the large-system limit.

For a photonic crystal, the states at the photonic band edges are propagating modes such that the electromagnetic field is distributed throughout the system. If the periodicity is disturbed, localized states begin to fill in the gap so that the states just below and just above become localized. Although the nature of the electromagnetic states in quasiperiodic structures needs to be further investigated, similar features are displayed by photonic quasicrystalline systems. In Figs. 5(a)-5(d) and 6(a)-6(d), we compare the azimuthal electric field distribution for modes below or above the band gap [plots (b) and (d) in Figs. 5 and 6], which display an extended character with the field distributed among many sites; and then modes at the band edges [plots (a) and (e) in Figs. 5 and 6], which show a high degree of spatial concentration. We also find that the formation of the TM band gaps is closely related to the formation of electromagnetic resonances localized within the dielectric cylinders [as shown by the (a) and (b) plots in Figs. 5 and 6] and that there is a strong correlation between the scattering properties of the individual scatterers (dielectric cylinders) and the band-gap
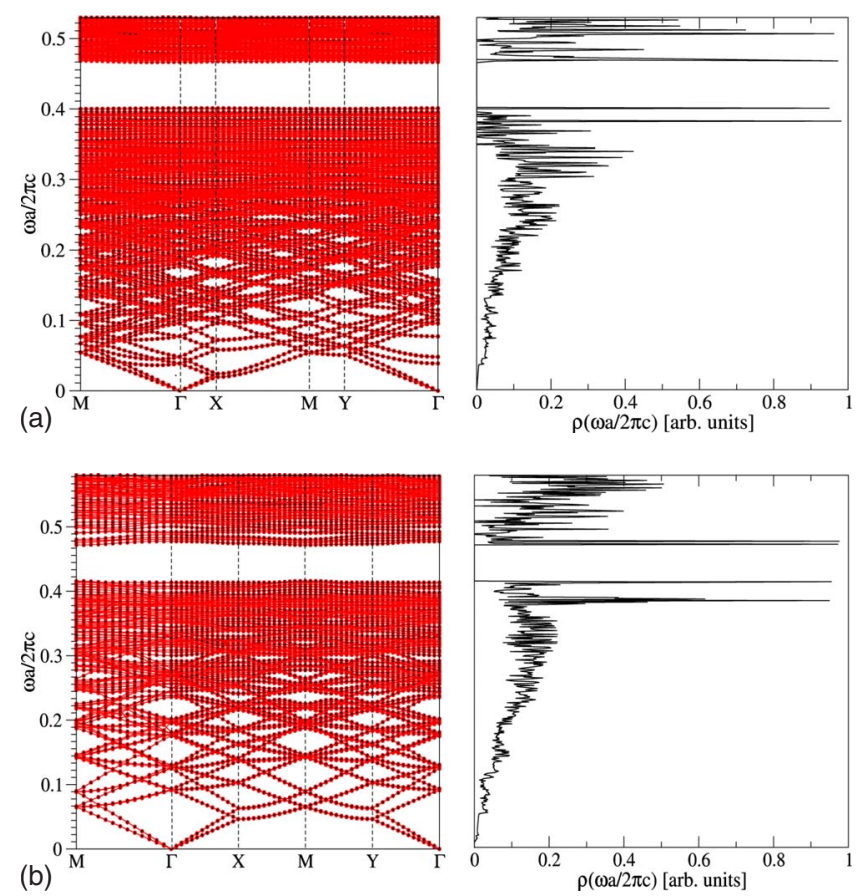

FIG. 4. (Color online) Photonic band structure and density of states for fivefold and eightfold symmetric photonic quasicrystals. (a) Photonic band structure and the corresponding density of states for a network constructed via the protocol in Sec. II from a 2/1 periodic approximant of the Penrose tiling with optimized cylinder radius $r / a=0.157$ and wall thickness $w / a=0.042$. The structure has a complete PBG of $15.14 \%$ with central frequency $\omega c /(2 \pi a)$ $=0.43$. (b) Photonic band structure and the corresponding density of states for a network constructed from a $3 / 2$ periodic approximant of the octagonal quasilattice with optimized cylinder radius $\mathrm{r} / \mathrm{a}$ $=0.1613$ and wall thickness $w / a=0.0136$. The structure has a complete PBG of $13 \%$ with central frequency $\omega c /(2 \pi a)=0.44$.

location. In particular, the largest TM gap occurs when the frequency of the first Mie resonance coincides with the lower edge of the photonic band gap. ${ }^{15}$ Analogous to the case of periodic systems, the electric field for the lower band-edge states is well localized in the cylinders (the high-dielectric component), thereby lowering their frequencies; and the electric field for the upper band-edge states are localized in the air fraction, increasing their frequencies. As shown in Figs. 5(e)-5(h) and 6(e)-6(h), an analogous behavior occurs for the azimuthal magnetic field distribution for TE modes: for states near the lower edge of the gap, the azimuthal magnetic field is mostly localized inside the air fraction and presents nodal planes that pass through the high index of refraction fraction of the structure while states near the upper edge display the opposite behavior.

\section{CONCLUSIONS}

In sum, we have shown that it is possible to expand the spectrum of dielectric materials with sizeable complete band gaps to include photonic quasicrystals by introducing a constrained optimization procedure. The quasicrystalline photonic structures can be manufactured using standard fabrication techniques used for photonic crystals. ${ }^{27}$ 


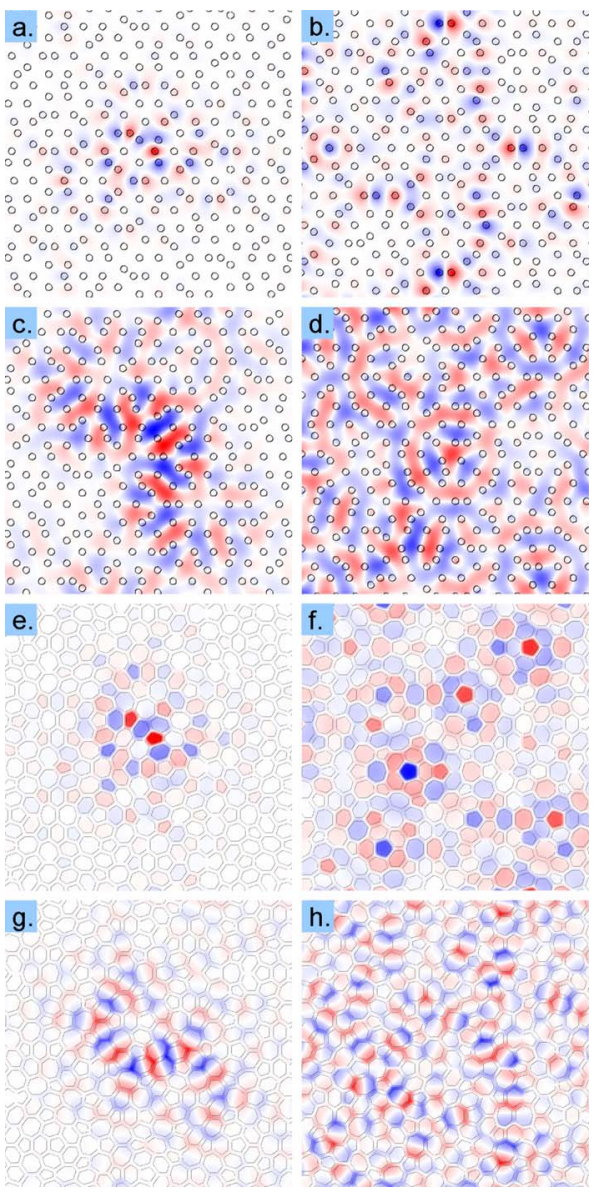

FIG. 5. (Color online) Panels (a)-(d) show the electric field distribution for a fivefold symmetric photonic quasicrystal optimized for TM polarized radiation. The structure consists of dielectric cylinders of radius $r / a=0.177$ placed at the vertices of a Penrose tiling and displays a TM photonic band gap of $36.5 \%$. Lower (a) and upper (c) band-edge modes display a well-defined degree of localization; modes just below the lower band edge (b) and just above the upper band edge (d) display an extended character. Panels (e)-(h) show the magnetic field distribution in fivefold symmetric quasicrystalline network optimized via the protocol in Sec. II for TE polarized radiation. The structure consists of trihedral network with wall thickness $w / a=0.102$ and displays a TE PBG of $42.5 \%$. The lower (e) and upper (g) band-edge modes display a high degree of spatial concentration and modes just below the lower band edge (f) and just above the upper band edge (h) display an extended character.

Photonic crystals, the quasicrystals considered here, and the disordered heterostructures discussed in Ref. 18 are all decorations of hyperuniform patterns. ${ }^{28}$ A point pattern is hyperuniform if the number variance within a spherical sampling window of radius $R$ (in $d$ dimensions) grows more slowly than the window volume for large $R$, i.e., $\left\langle N_{R}^{2}\right\rangle$ $-\left\langle N_{R}\right\rangle^{2}=A R^{p}$, where $p<d$. Two dimensional crystal and quasicrystal patterns both correspond to $p=1$. Our conjecture in Ref. 18, which was supported by a variety of examples, stated that a higher degree of hyperuniformity (smaller coefficient $A$ ) is advantageous for obtaining substantial complete PBGs. In the Appendix, we provide the first calculations of the coefficient $A$ for two-dimensional quasicrystals, namely,

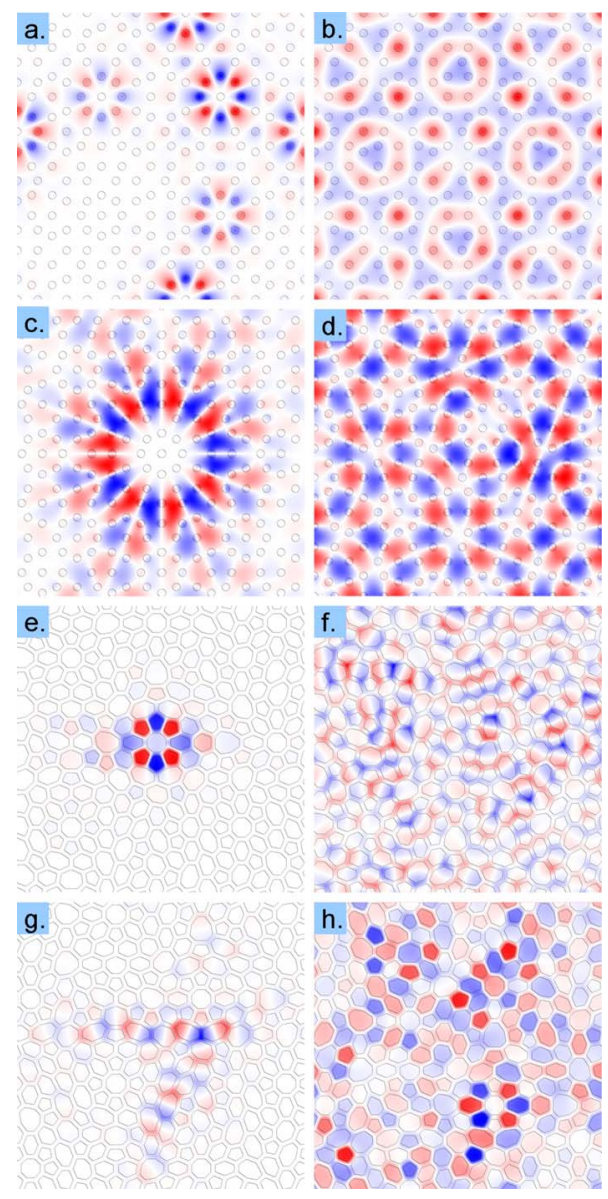

FIG. 6. (Color online) Panels (a)-(d) show the electric field distribution in eightfold symmetric structures for TM-polarized radiation. The structure consists of dielectric cylinders of radius $r / a$ $=0.189$ and displays a TM PBG of $42 \%$. Lower (a) and upper (c) band-edge modes displaying a well-defined degree of localization; modes just below the lower band edge (a) and just above the upper band edge (d) displaying an extended character. Panels (e)-(h) show analogous magnetic field distributions in eightfold symmetric heterostructures optimized via the protocol in Sec. II for TE-polarized radiation. The structure consists of trihedral network with wall thickness $w / a=0.101$ and displays a TE PBG of $40 \%$. Lower (e) and upper $(\mathrm{g})$ band-edge modes display a well-defined degree of spatial concentration; modes just below the lower band edge (f) and just above the upper band edge (h) display an extended character.

the fivefold and eightfold examples considered here. The number variance for quasicrystals is greater than for crystals (for the same density) and less than the variance of disordered heterostructures. The results here are consistent with our conjecture in that the width of the optimal complete band gaps for the photonic quasicrystals lies between the optima found for crystals and disordered heterostructures.

Although photonic crystals have slightly larger complete band gaps, quasicrystalline PBG materials offer advantages for many applications. In the case of quasicrystalline structures the PBGs are significantly more isotropic, which is advantageous for use as highly efficient isotropic thermal radiation sources. ${ }^{29}$ The properties of defects and channels useful for controlling the flow of light are different for crystal and quasicrystal structures. Radiation with frequencies 
above or below the band edges are propagating modes that are transmitted through photonic crystals but are more localized modes in the case of quasiperiodic patterns, which give the former advantages in some applications, such as light sources. ${ }^{30}$ On the other hand, due to the lack of translational symmetry, PBG structures constructed around quasiperiodic point patterns can provide a large number of inequivalent local environments and as such can support a rich variety of localized modes. These localized modes can be employed to design laser systems with highly unusual field patterns with possible applications to biological sensing. ${ }^{31}$

\section{ACKNOWLEDGMENT}

This work was supported by the National Science Foundation under Grant No. DMR-0606415.

\section{APPENDIX: ASYMPTOTIC NUMBER VARIANCE FOR THE FIVEFOLD AND EIGHTFOLD QUASICRYSTAL POINT PATTERNS}

Here we compute the asymptotic number variance for the fivefold and eightfold quasicrystal point patterns in two dimensions obtained from the Penrose and octagonal tilings. For such point patterns, the number variance for large windows grows like the perimeter of the window, i.e.,

$$
\left\langle N_{R}^{2}\right\rangle-\left\langle N_{R}\right\rangle^{2}=A \frac{R}{D}+\mathcal{O}(1), \quad R \rightarrow \infty,
$$

where $R$ is the window radius and $D$ is the mean-nearestneighbor distance associated with the point pattern. In order to compare the dimensionless surface area coefficient $A$ for different point patterns, the comparison must be made at the same number density $\rho$ (number of points per unit volume). It was shown in Ref. 28 that this amounts to rescaling $A$ such that $A / \sqrt{\phi}$, where

$$
\phi=\rho \frac{\pi}{4} D^{2}
$$

is an effective packing fraction. Thus, we define the rescaled coefficient $\widetilde{A} \equiv A / \sqrt{\phi}$.

The rescaled surface-area coefficients can be evaluated numerically by employing finite large quasicrystalline point patterns (of around 15000 points) and count the number of points in a circular window of radius $R$. For a fixed radius, the circular window scans the quasicrystalline domain and the variance of the number of points in the window is evaluated (the windows employed in our calculations contain from a few to 5000 points and to calculate the variance we use 1000 configurations with a fixed window size). The surfacearea coefficient is then obtained by analyzing the behavior of the number variance in the limit of large window size.

We find that the rescaled surface-area coefficients for the fivefold and eightfold cases are given, respectively, by $\widetilde{A}_{\text {fivefold }}=0.60052$ and $\tilde{A}_{\text {eightfold }}=0.59567$. Our results for these quasicrystal cases should be compared to results for some periodic point patterns; for the triangular, square, honeycomb and Kagomé lattices, $\widetilde{A}_{\text {triangular }}=0.508347, \widetilde{A}_{\text {square }}$ $=0.51640, \quad \widetilde{A}_{\text {honeycomb }}=0.56702$, and $\tilde{A}_{\text {Kagome }}=0.58699$, respectively. ${ }^{28}$ It is noteworthy that the triangular lattice has been proven to have the smallest surface-area coefficient among all lattices and is thought to be the global minimum among all point patterns. Thus, we see that the quasicrystal point patterns studied here have a high degree of hyperuniformity, even if not as large as that for the aforementioned crystals. These calculations of the surface-area coefficients and PBGs reported here are consistent with the conjecture made in Ref. 18, namely, that the width of complete PBGs of high-dielectric contrast photonic structures is correlated with the degree of hyperuniformity of the underlying point pattern.
*Electronic address: florescu@ princeton.edu

${ }^{1}$ S. John, Phys. Rev. Lett. 58, 2486 (1987).

${ }^{2}$ E. Yablonovitch, Phys. Rev. Lett. 58, 2059 (1987).

${ }^{3}$ H. Altug, D. Englund, and H. Vuckovic, Nat. Phys. 2, 484 (2006).

${ }^{4}$ J.-M. Lourtioz, H. Benisty, V. Berger, J. M. Gerard, D. Maystre, and A. Tchelnokov, Photonic Crystals, Towards Nanoscale Photonic Devices, 2nd ed. (Springer, Heidelberg, 2008).

${ }^{5}$ I. EI-Kady, M. M. R. Taha, and M. F. Su, Appl. Phys. Lett. 88, 253109 (2006).

${ }^{6}$ A. Chutinan, S. John, and O. Toader, Phys. Rev. Lett. 90, 123901 (2003); M. Florescu and S. John, Phys. Rev. A 69, 053810 (2004).

${ }^{7}$ S. John and J. Wang, Phys. Rev. B 43, 12772 (1991).

${ }^{8}$ D. Levine and P. J. Steinhardt, Phys. Rev. Lett. 53, 2477 (1984).

${ }^{9}$ Y. S. Chan, C. T. Chan, and Z. Y. Liu, Phys. Rev. Lett. 80, 956 (1998).

${ }^{10}$ M. E. Zoorob, M. D. B. Charlton, G. J. Parker, J. J. Baumberg, and M. C. Netti, Nature (London) 404, 740 (2000).
${ }^{11}$ M. Bayindir, E. Cubukcu, I. Bulu, and E. Ozbay, Phys. Rev. B 63, 161104(R) (2001).

${ }^{12}$ X. Zhang, Z-Q Zhang and C. T. Chan, Phys. Rev. B 63, 081105(R) (2001).

${ }^{13}$ K. Wang, S. David, A. Chelnokov, and J. M. Lourtioz, J. Mod. Opt. 50, 2095 (2003).

${ }^{14}$ A. Della Villa, S. Enoch, G. Tayeb, V. Pierro, V. Galdi, and F. Capolino, Phys. Rev. Lett. 94, 183903 (2005).

${ }^{15}$ C. Rockstuhl and U. Peschel, and F. Lederer, Opt. Lett. 31, 1741 (2006).

${ }^{16}$ D. N. Chigrin and A. V. Lavrinenko, in Metamaterials Handbook, edited by F. Capolino (CRC, Boca Raton, 2009), Vol. II.

${ }^{17}$ R. D. Meade, A. M. Rappe, K. D. Brommer, and J. D. Joannopoulos, J. Opt. Soc. Am. B 10, 328 (1993).

${ }^{18} \mathrm{M}$. Florescu, S. Torquato, and P. J. Steinhardt (unpublished).

${ }^{19}$ R. Penrose, Bull. Inst. Math. Appl. 10, 266 (1974).

${ }^{20}$ B. Grunbaum, Z. Grunbaum, and G. Shephard, Comput. Math. Appl. 12, 641 (1986).

${ }^{21}$ M. Duneau and A. Katz, Phys. Rev. Lett. 54, 2688 (1985). 
${ }^{22}$ The Physics of Quasicrystals, edited by P. J. Steinhardt and S. Ostlund (World Scientific, Singapore, 1987).

${ }^{23}$ C. Kao, S. Osher, and E. Yablonovitch, Appl. Phys. B: Lasers Opt. 81, 235 (2005).

${ }^{24}$ O. Sigmund and K. Hougaard, Phys. Rev. Lett. 100, 153904 (2008).

${ }^{25}$ M. C. Rechtsman, H. C. Jeong, P. M. Chaikin, S. Torquato, and P. J. Steinhardt, Phys. Rev. Lett. 101, 073902 (2008).

${ }^{26}$ S. G. Johnson and J. D. Joannopoulos, Opt. Express 8, 173
(2001).

${ }^{27}$ H. Fu, Y. Chen, R. Chen, and C. Chang, Opt. Express 13, 7854 (2005).

${ }^{28}$ S. Torquato and F. H. Stillinger, Phys. Rev. E 68, 041113 (2003).

${ }^{29}$ M. Florescu, K. Busch and J. P. Dowling, Phys. Rev. B 75, 201101(R) (2007).

${ }^{30}$ M. Notomi, H. Suzuki, T. Tamamura, and K. Edagawa, Phys. Rev. Lett. 92, 123906 (2004).

${ }^{31}$ S. Boriskina and L. Dal Negro, Opt. Express 16, 12511 (2008). 